\title{
Correction to: Heritable and non-heritable uncommon causes of stroke
}

\author{
A. Bersano ${ }^{1} \cdot$ M. Kraemer ${ }^{2,3} \cdot$ A. Burlina ${ }^{4} \cdot$ M. Mancuso ${ }^{5} \cdot$ J. Finsterer $^{6} \cdot$ S. Sacco ${ }^{7}$. C. Salvarani ${ }^{8} \cdot$ L. Caputi ${ }^{1}$. \\ H. Chabriat ${ }^{9} \cdot$ S. Lesnik Oberstein ${ }^{10} \cdot$ A. Federico ${ }^{11} \cdot$ E. Tournier-Lasserve ${ }^{12} \cdot$ D. Hunt ${ }^{13} \cdot$ M. Dichgans $^{14} \cdot$ M. Arnold $^{15}$. \\ S. Debette ${ }^{16} \cdot$ H. S. Markus ${ }^{17}$
}

Published online: 18 June 2020

(c) Springer-Verlag GmbH Germany, part of Springer Nature 2020

\section{Correction to: Journal of Neurology https://doi.org/10.1007/s00415-020-09836-x}

The original version of this article unfortunately contained a mistake. Figure 3 caption is incorrect and co-author name should be E.Tournier-Lasserve.

The correct Fig. 3 caption with figure is placed in the following page.

The original article can be found online at https://doi.org/10.1007/ s00415-020-09836-x.

\section{A. Bersano}

anna.bersano@gmail.com

1 Cerebrovascular Unit, Fondazione IRCCS Istituto Neurologico Carlo Besta, Milan, Italy

2 Department of Neurology Alfried, Krupp-Hospital, Essen, Germany

3 Department of Neurology, Medical Faculty, Heinrich-Heine-University, Düsseldorf, Germany

4 Neurological Unit, St. Bassano Hospital, Bassano del Grappa, Italy

5 Department of Clinical and Experimental Medicine, Neurological Institute, University of Pisa, Pisa, Italy

6 Krankenanstalt Rudolfstiftung, Messerli Institute, Vienna, Austria

7 Department of Neurology, Avezzano Hospital, University of L'Aquila, L'Aquila, Italy

8 University of Modena and Reggio Emilia, and Azienda USL-IRCCS, Reggio Emilia, Italy

9 Department of Neurology and CERVCO, DHU Neurovasc, INSERM U1141, University of Paris, Paris, France
10 Department of Clinical Genetics, Leiden University Medical Center, Leiden, The Netherlands

11 Department of Medicine, Surgery and Neurosciences, University of Siena, Siena, Italy

12 Department of Genetics, Lariboisière Hospital and INSERM U1141, Paris-Diderot University, Paris, France

13 MRC Human Genetics Unit, Institute of Genetics and Molecular Medicine, University of Edinburgh, Edinburgh EH4 2XU, UK

14 Institute for Stroke and Dementia Research, Klinikum Der Universität München, Munich, Germany

15 Inserm Centre Bordeaux Population Health (U1219), University of Bordeaux, Bordeaux, France

16 Department of Neurology, INSELSPITAL, University Hospital Bern, Bern, Switzerland

17 Stroke Research Group, Department of Clinical Neurosciences, University of Cambridge, Cambridge, UK 


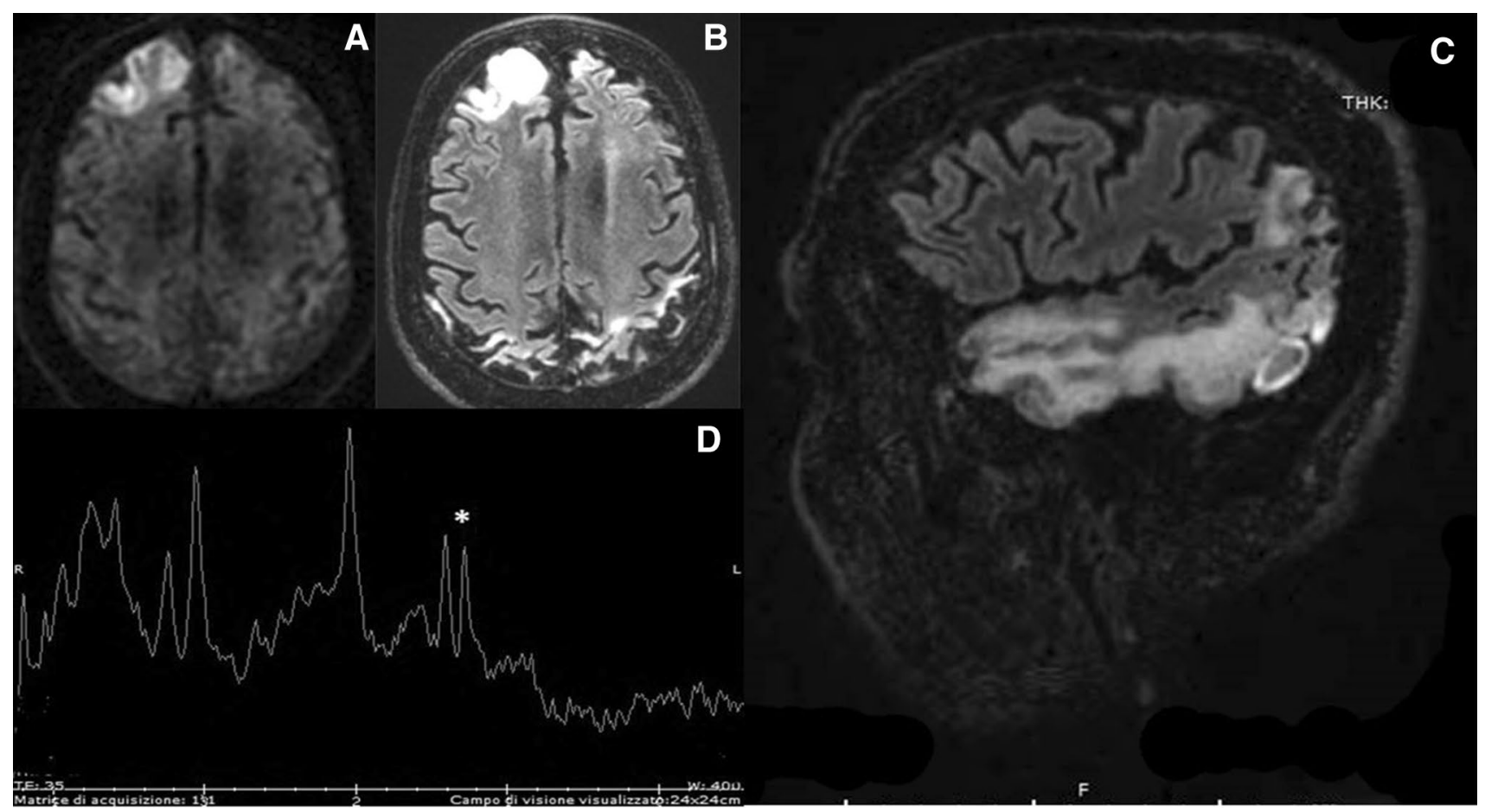

Fig. 3 Cranial magnetic resonance findings of stroke-like episodes in a young man that do not respect the vascular territories of major cerebral arteries or border zones. DWI (a) and FLAIR (b) sequences reveal an acute stroke-like lesion in the right frontal lobe, with an older bigger stroke-like lesion in the right temporal lobe also documented (c). The asterisk (d) shows lactate peak at the proton spectroscopy 